\title{
Elevated Preoperative Serum CEA Level Is Associated with Poor Prognosis in Patients with Hepatocellular Carcinoma Through the Epithelial-Mesenchymal Transition
}

\author{
MASATO YOSHIKAWA, YUJI MORINE, TETSUYA IKEMOTO, SATORU IMURA, JUN HIGASHIJIMA, \\ SHUICHI IWAHASHI, YU SAITO, CHIE TAKASU, SHINICHIRO YAMADA, DAICHI ISHIKAWA, \\ HIROKI TERAOKU, ATSUSHI TAKATA, TOSHIAKI YOSHIMOTO and MITSUO SHIMADA \\ Department of DigestiveSurgery, Institute of Health Bioscience, \\ The University of Tokushima Graduate School, Tokushima, Japan
}

\begin{abstract}
Background: Serum carcinoembryonic antigen (CEA) is used as an indicator of tumor progression in a variety of carcinomas. A subset of patients with hepatocellular carcinoma (HCC) exhibit increased serum CEA level, but the significance of this is unclear. In this study, we investigated the prognosis of patients with HCC with increased serum CEA, and explored the correlations with expression of carcinoembryonic antigen-related cell adhesion molecule 1 (CEACAM1) and epithelial-mesenchymal transition (EMT) and tumor angiogenesis. Materials and Methods: One hundred and twenty-three patients with HCC who underwent radical resection were divided into two groups according to a cut-off value of $5.0 \mathrm{ng} / \mathrm{ml}$ for serum CEA: high $(n=24)$ and normal $(n=99)$ groups. We compared the clinicopathological factors with serum CEA levels and its correlations with CEACAM1 expression, EMT-related factors and microvessel density (MVD) of tumor tissues by immunohistochemistry. Results: In the high CEA group, the disease-free survival (DFS) rate was significantly worse than in the normal CEA group. Multivariate analysis revealed that a high CEA level was an independent factor predictive of recurrence. Furthermore, increased serum CEA levels were positively correlated with CEACAM1 expression. Moreover, CEACAM1 expression was positively correlated with expression of EMT-related factors and MVD of tumor tissues. Conclusion: Increased serum CEA level reflected CEACAMI
\end{abstract}

Correspondence to: Professor Mitsuo Shimada, MD, Ph.D., Department of Surgery, Institute of Biomedical Sciences, Tokushima University Graduate School, 3-18-15 Kuramoto-cho, Tokushima 770-8503, Japan. Tel: +81 886339276, Fax: +81 886319698 ,e-mail: mitsuo.shimada@tokushima-u.ac.jp

Key Words: CEA, CEACAM, epithelial mesenchymal transition, hepatocellular carcinoma. expression and was an independent factor predictive of recurrence in HCC through EMT and tumor angiogenesis.

Hepatocellular carcinoma (HCC) is one of the leading causes of cancer-related deaths worldwide. Despite improvements in treatment modalities, including surgical resection, radiofrequency ablation, trans-arterial therapy and sorafenib, long-term survival remains dismal, partly because of posttreatment relapse and distant metastasis $(1,2)$. Therefore, it is of great importance to identify novel prognostic markers of HCC, and further improve diagnostic tools for tumor relapse in patients with $\mathrm{HCC}$.

Serum carcinoembryonic antigen (CEA) is increased in various types of adenocarcinoma, such as colon and gastric cancer, and is associated with tumor progression. However, in $\mathrm{HCC}$, the level of serum CEA is not a specific marker for carcinogenesis or recurrence after curative treatment. Nonetheless, some subsets of HCC demonstrate aggressive malignant behavior with elevated serum CEA level, although the significance of this remains unknown.

In recent years, the CEA family of immunoglobulinrelated proteins was classified into several subtypes, known as carcinoembryonic antigen-related cell adhesion molecules (CEACAMs). The CEACAMs are encoded by 12 genes, and show distinct expression patterns in different cell types (35). CEACAM subtypes 1,5 , and 6 are described to be underor overexpressed in several tumor types including lung, colon, and pancreatic cancer, and melanoma (6-11). In particular, CEACAM1 was found to induce tumor invasion and transition, and its expression is important for malignant behavior in HCC (12-14). The epithelial-mesenchymal transition (EMT) plays an important role in tumor malignancy including metastasis and invasion. In HCC, it has been reported that EMT stimulated tumor recurrence through the transforming growth factor $\beta$ (TGF $\beta$ )/SMAD transcriptional pathway (15). 
In this study, we investigated whether an increased serum CEA level reflects CEACAM1 expression, and induces EMT and angiogenesis to promote the malignant behavior of HCC.

\section{Materials and Methods}

Patients and surgical procedures. One hundred twenty-three patients with HCC who underwent radical resection at Tokushima University Hospital, Japan, between April 2005 and March 2012, were enrolled. All 123 patients underwent liver resections for initial treatment of HCC, and the curative resections were confirmed by pathological examination. Cases with mixed type liver cancer, including intrahepatic cholangiocarcinoma, were excluded from this analysis by pathologist. Staging and curability were defined according to the Classification of Primary Liver Cancer by the Liver Cancer Study Group of Japan (16). There were 91 men and 32 women, ranging from 33 to 84 years of age, with a mean age of 66 years. Clinicopathological characteristics, including age, sex, liver function, hepatitis and pathological tumor status, were available for all patients. This study was approved by the Ethics Committee of our hospital (no.2345; Tokushima clinical trial system), and written informed consent for the use of resected tissues was obtained from all patients. Preoperative serum CEA levels were measured and patients were divided into two groups (high and normal CEA groups) using a cut-off value of $5.0 \mathrm{ng} / \mathrm{ml}$ (upper limit of normal range) to evaluate relationships with clinicopathological factors and prognosis.

Patient follow-up. We followed-up all patients in the outpatient clinic according to a standard protocol (22). Briefly, patients were followed-up every 2 months during the first year after surgery and at least every 3-4 months thereafter. Both $\alpha$-fetoprotein (AFP) and protein-induced vitamin $\mathrm{K}$ absence or antagonist-II (PIVKA-II) were evaluated each time. Abdominal dynamic computed tomography was performed every 6 months. A diagnosis of recurrence was made based on the typical imaging appearance in computed tomography or magnetic resonance imaging (23-25). Intrahepatic recurrence of HCC was diagnosed when the following two criteria were met: the lesion was enhanced during the arterial phase and was hypoattenuating or hypointense relative to the surrounding liver during the venous or delayed phases. Additional imaging findings regarded as suggestive but not diagnostic of intrahepatic recurrence of HCC included lesions that had arterial enhancement or were hypoattenuating or hypointense relative to the surrounding liver during the venous or delayed phases, had peripheral rim enhancement (suggesting a capsule or pseudocapsule) during the delayed phase, showed decreased signal intensity during the liver-specific hepatobiliary phase, or showed moderately increased signal intensity on T2-weighted magnetic resonance imaging. An elevated level of AFP or PIVKA-II was also taken into consideration for confirmation of recurrence.

Immunohistochemistry. For each patient, we chose the most typical hematoxylin and eosin (H\&E)-stained tumor slide for immunostaining. Specimens were fixed in $10 \%$ formaldehyde, embedded in paraffin, and cut into $4 \mu \mathrm{m}$-thick sections. Immunostaining was performed as previously reported (17-21). Sections were deparaffinized with xylene, followed by rehydration in an ethanolic series. Sections were treated with $3 \%$ hydrogen peroxide in methanol for $10 \mathrm{~min}$ to block endogenous peroxidase activity. Antigen retrieval was performed by boiling in $10 \mathrm{mM}$ citrate buffer ( $\mathrm{pH}$ ) using a microwave. After incubation with $1 \%$ bovine serum albumin to block nonspecific antibody binding, sections were incubated with primary antibodies against CEACAM1 (1:100; ab49510; Abcam, Cambridge, UK), E-cadherin (1:50; M3612; Dako, Tokyo, Japan), vimentin (undiluted solution; IR630; Dako), and CD34 (1:50; M7165; Dako) for $60 \mathrm{~min}$ at room temperature. After washing with phosphate-buffered saline, sections were subjected to the Dako REAL EnVision/HRP detection system (Dako) for $60 \mathrm{~min}$ at room temperature. The peroxidase reaction was developed with 3,3'-diaminobenzidine as the chromogen. Sections were counter-stained with $10 \%$ Mayer's hematoxylin, dehydrated in a graded series of ethanol, treated with xylene, and mounted in a synthetic resin.

Immunohistochemical evaluations were performed by a pathologist who was blinded to information for each patient.

Immunohistochemical staining and assessment. CEACAM1: To evaluate CEACAM1 expression, the staining score was semiquantitatively calculated by the percentage of stained areas and intensities of tumor tissues. CEACAM1 expression was scored by intensity (1-3+), area (\%) in cytoplasm, and grade: low: $1+,<70 \%$ or $2+,<30 \%$; high: $1+, \geq 70 \%$ or $2+, \geq 30 \%$ or $3+$, any (6).

E-Cadherin and vimentin: To evaluate E-cadherin and vimentin expression, the staining score was semiquantitatively calculated by the percentage of stained areas of tumor tissues. E-Cadherin expression was scored by staining area: grade 1,0-25\%; grade 2 , 26-50\%; grade 3, $>51 \%$. Vimentin expression was scored by staining area: grade 1,0-25\%; grade 2, $>26 \%$ (19).

CD34: To determine CD34 expression, after selecting the best five fields at $\times 40$ the field of view, microvessels with CD34 staining were counted at $\times 200$ the field of view, and their average value was defined as the MVD $(18,20)$.

Statistical analysis. All statistical analyses were performed using JMP 10.0.2 statistical software (SAS, Cary, NC, USA). All results are expressed as the mean \pm standard error. The Mann-Whitney test was used to compare continuous variables. The chi-squared test was applied for categorical data. The patient survival rate after 5 years from surgery was calculated by the Kaplan-Meier product limit method. Differences in survival between groups were compared using the log-rank test. Prognostic factors were examined using univariate and multivariate analyses (Cox proportional hazards regression model). Continuous variables were generally classified into two groups according to the median value of each variable. A value of $p<0.05$ was considered statistically significant.

\section{Results}

Serum CEA and clinicopathological features. The patients were divided into two groups: high $(n=24)$ and normal $(n=99)$ according to serum CEA expression $(5.0 \mathrm{ng} / \mathrm{ml}$ cutoff). In the analysis of CEA level, there was a significantly higher number of patients with hepatitis $\mathrm{C}$, however, no other significant differences in clinicopathological and tumor-related factors, such as age, liver function, tumor marker, were identified between the high and normal CEA groups (Table I). 
Table I. Clinicopathological factors according to serum carcinoembryonic antigen (CEA) level.

\begin{tabular}{lccc}
\hline Factors & \multicolumn{2}{c}{ Serum CEA level } & \multirow{2}{*}{$p$-Value } \\
\cline { 2 - 3 } & $\begin{array}{c}\text { Normal } \\
(\mathrm{n}=99)\end{array}$ & $\begin{array}{c}\text { High } \\
(\mathrm{n}=24)\end{array}$ & \\
\hline Age (years) & $66.2 \pm 1.1$ & $66.8 \pm 2.2$ & 0.81 \\
Sex: Male/female & $72 / 27$ & $19 / 5$ & 0.51 \\
HBs Ag: -/+ & $75 / 24$ & $18 / 6$ & 0.94 \\
HCV Ab: -/+ & $60 / 39$ & $8 / 16$ & 0.02 \\
Child Pugh: A/B, C & $92 / 7$ & $23 / 1$ & 0.59 \\
T-Bil (mg/dl) & $0.9 \pm 0.1$ & $0.9 \pm 0.1$ & 0.74 \\
Alb (g/dl) & $3.8 \pm 0.1$ & $3.8 \pm 0.1$ & 0.87 \\
ICGR 15 (\%) & $14.2 \pm 1.1$ & $16.4 \pm 2.2$ & 0.38 \\
Tumor number (single/multiple) & $74 / 25$ & $18 / 6$ & 0.98 \\
Tumor size (cm) & $5.2 \pm 0.4$ & $3.8 \pm 0.7$ & 0.09 \\
vv: -/+ & $77 / 22$ & $16 / 8$ & 0.27 \\
vp: -/+ & $77 / 22$ & $17 / 7$ & 0.48 \\
im: -/+ & $89 / 10$ & $22 / 2$ & 0.76 \\
Differentiation: Well/mod, poor & $15 / 84$ & $3 / 21$ & 0.74 \\
Stage: I, II/III, IV & $61 / 39$ & $15 / 8$ & 0.60 \\
\hline
\end{tabular}

HBs Ag: Hepatitis B virus antigen, HCV Ab: hepatitis C virus antibody, T-Bil: total bilirubin, Alb: albumin, ICGR 15: indocyanine green retention test at $15 \mathrm{~min}, \mathrm{vv}$ : venous invasion, vp: port vein invasion, im: intrahepatic metastasis.

Serum CEA and overall survival/disease-free survival rates. For overall survival, there was no significant difference between the high and normal CEA groups (5-year survival rate: $70.8 \%$ vs. $68.8 \%$, respectively, $p=0.33$ ) (Figure $1 \mathrm{~A}$ ). In disease-free survival, the high CEA group had significantly worse prognosis compared to the normal CEA group (5-year disease-free survival rate: $16.0 \%$ vs. $66.5 \%$, respectively, $p<0.01$ ) (Figure 1B).

In univariate analysis of risk factors for tumor recurrence, the presence of multiple tumors, high preoperative PIVKAII level $(>400 \mathrm{mAU} / \mathrm{ml})$ and high preoperative carbohydrate antigen 19-9 (CA19-9) level (>37.0 ng/ml) were determined to be significant risk factors for tumor recurrence, along with high CEA level (Table II). In multivariate analysis, high CEA level (hazard ratio $=4.40,95 \%$ confidence interval $=2.24-8.46$, $p<0.01$ ), and multiple tumors (hazard ratio $=2.27,95 \%$ confidence interval $=1.16-4.36, p=0.02$ ) were identified as independent factors predictive of recurrence (Table II).

Correlation between elevated serum CEA level and CEACAM1 expression. Firstly, regarding overall survival, there was no significant difference between the high and low CEACAM1 groups (5-year survival rate: $80.4 \%$ vs. $83.4 \%$, respectively, $p=0.27$ ) (Figure $1 \mathrm{C}$ ). In disease-free survival, the high CEACAM1 group had significantly worse prognosis compared with the low CEACAM1 group (5-year diseasefree survival rate: $29.7 \%$ vs. low $58.1 \%$, respectively, $p=0.04$ ) (Figure 1D). Next, we examined the correlation between tumor CEA level and CEACAM1 expression. Representative staining of CEACAM1 according to each staining score is shown in Figure 2A. CEACAM1 expression was found to be significantly higher in the high CEA group than in the normal CEA group $(p<0.01)$ (Table III).

Correlation between expression of CEACAM1 and EMTrelated factors and tumor angiogenesis. To confirm whether CEACAM1 expression regulates EMT and tumor angiogenesis, we examined the correlations between expression of CEACAM1 and E-cadherin, vimentin and CD34. Figure 2 shows representative staining of E-cadherin, vimentin and CD34. CEACAM1 expression was inversely correlated with E-cadherin expression $(p<0.05)$, and was positively correlated with vimentin expression $(p=0.06)$ (Table IV). In addition, the MVD of the low $(n=69)$ CEACAM1 group was and significantly lower than that of the high CEACAM1 group $(\mathrm{n}=54)(p<0.01)$ (Table IV). There was a significant positive correlation between CEACAM1 expression and MVD $(p<0.01)$.

\section{Discussion}

CEA is a glycoprotein comprised of 50-60\% sugar chains with a molecular weight of approximately $180,000 \mathrm{Da}$ and was first revealed to be expressed in human colon cancer tissues and in fetal gut (26). CEA is increased in carcinomas of endodermal origin, particularly in adenocarcinomas. In addition, CEA is also known to act as a cell adhesion factor, and is increased in the bloodstream as a result of a weakness of cell adhesion during tumor growth, a known mechanism of increasing CEA level (27). In previous reports, Lens culinaris agglutinin reactive $\alpha$-fetoprotein isoform (AFP-L3) (28) and PIVKA-II (29), specific HCC markers, have been reported as postoperative recurrence prognostic factors. However, there are few reports on the relationship between postoperative recurrence of HCC and the CEA level, despite its status as the most widely used tumor marker. Meanwhile, some subsets of HCC demonstrate aggressive malignant behavior with elevated serum CEA level, although the significance remains unclear.

Recently a number of subtypes of CEACAMs have been identified, and CEACAM1, 5, 6 were shown to be factors predictive of recurrence in pancreatic cancer (6). Furthermore, CEACAM1 expression was associated with reduced survival and metastasis in lung cancer (21), and the relationship between CEACAMs and tumor malignancy have been reported in various cancer types, such as colon cancer and malignant melanoma $(30,31)$. In HCC, although the relationship between CEACAM1 expression and tumor malignancy is controversial, several reports indicated that CEACAM1 expression is associated with tumor malignancy by changing the isoform balance 

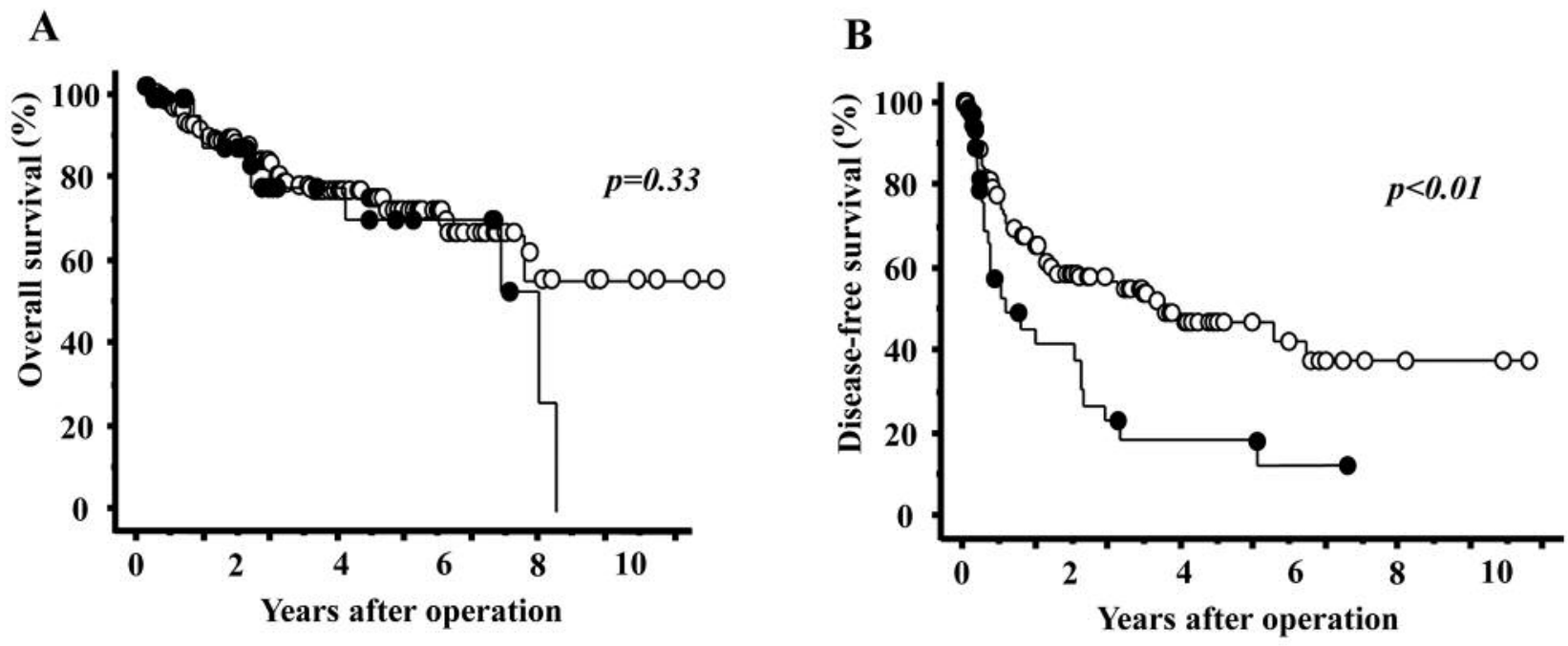

C

D
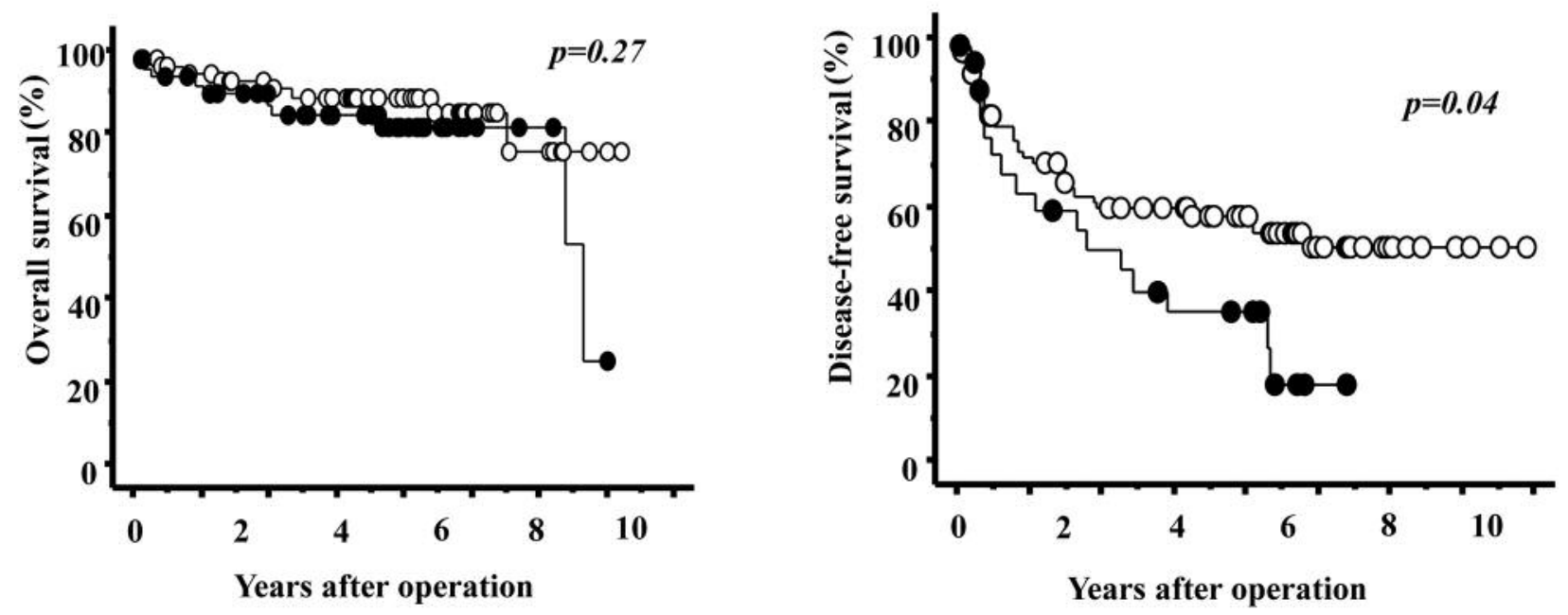

Figure 1. Kaplan-Meier overall $(A, C)$ and disease-free $(B, D)$ survival curves for patients according to low (open symbols) and high (closed symbols) expression of carcinoembryonic antigen (CEA) (A, B) and carcinoembryonic antigen-related cell adhesion molecule 1 (CEACAM1) (C, D). Low CEA group: $n=99$, high CEA group: $n=24$; low CEACAM1 group: $n=69$, high CEACAM1 group: $n=54$.

of CEACAM1-L and S (13) and subcellular CEACAM1 expression localization $(14,32)$.

In our study, patients with HCC with high cytoplasmic CEACAM1 expression had significantly worse prognosis, and expression of E-cadherin, a down-regulator of EMT, was significantly reduced, while vimentin, an up-regulator of EMT, had a tendency to increase. Moreover, tumor MVD of high CEACAM1-expressing patients was significantly increased. This suggests that CEACAM1 induces HCC malignancy through EMT and angiogenesis.

It is unclear whether serum CEA exerts any effects in the tumor microenvironment; however, a recent report indicated that CEA-expressing cells obtain malignant behavior, such as increased proliferation, and exhibit tumor angiogenesis and invasion through endothelial cell activation in vitro (33). This reveals that increased serum CEA is not just a biomarker of tumor progression, but also an upstream factor in a mechanism of tumor malignancy.

In conclusion, the preoperative serum CEA level significantly reflects CEACAM1 expression, and CEACAM1 appears to induce EMT and tumor angiogenesis. This suggests that serum CEA may be a reliable biomarker of recurrent HCC. To our knowledge, this is the first report to demonstrate the relationship between postoperative recurrence of HCC and preoperative serum CEA level, and the mechanism of recurrence through EMT and angiogenesis. 
A

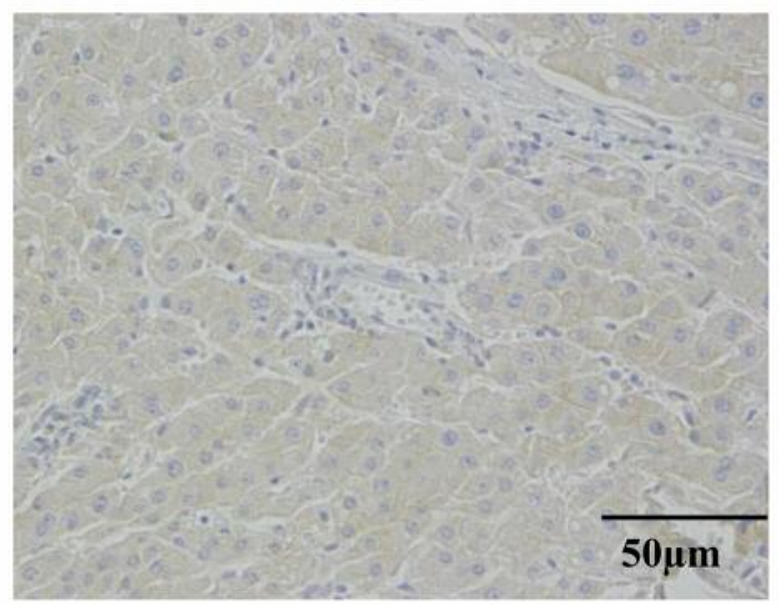

B

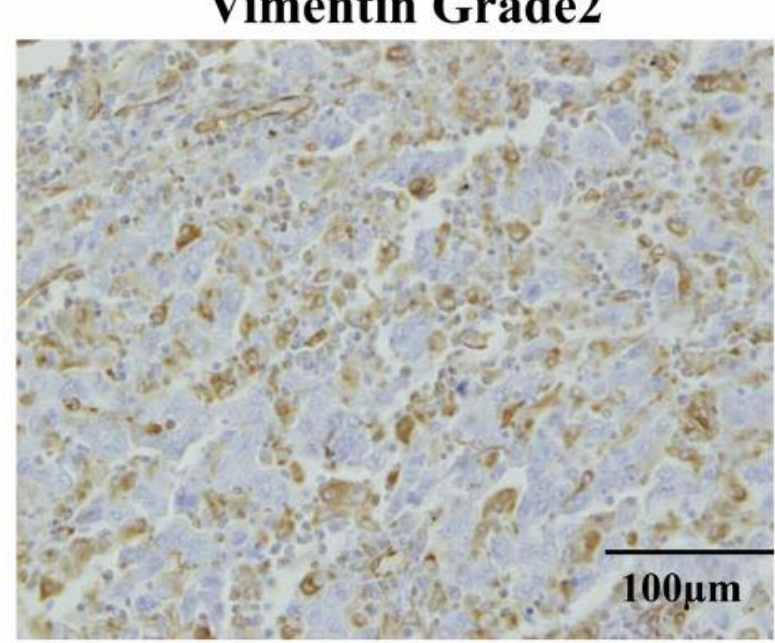

High

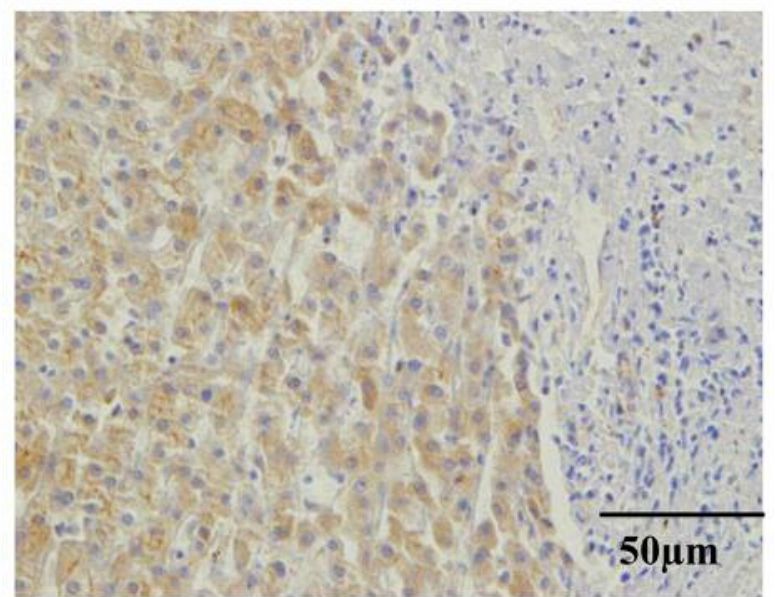

E-Cadherin Grade3

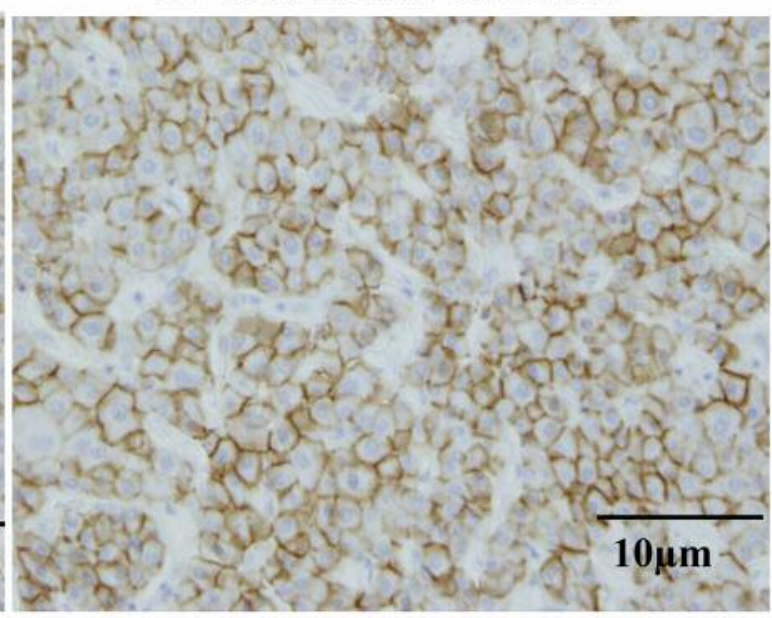

MVD High

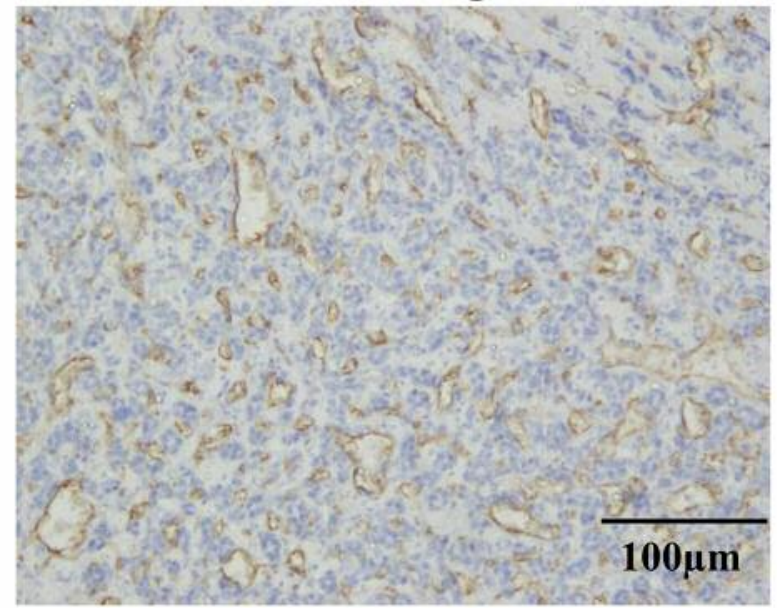

Figure 2. Representative staining of according to staining score (A), and representative staining of E-cadherin, vimentin and CD34 for microvessel density $(M V D)$. 
Table II. Predictive factors for disease-free survival (DFS).

\begin{tabular}{|c|c|c|c|c|}
\hline \multirow[t]{2}{*}{ Factor } & \multicolumn{2}{|c|}{ Univariate } & \multicolumn{2}{|c|}{ Multivariate } \\
\hline & 5-Year DFS (\%) & $p$-Value & HR $(95 \% \mathrm{CI})$ & $p$-Value \\
\hline Age: $\leq 65 />65$ Years & $61.2 / 47.1$ & 0.76 & & \\
\hline Gender: Male/female & $50.6 / 56.4$ & 0.14 & & \\
\hline HBs Ag: $-/+$ & $55.1 / 52.7$ & 0.72 & & \\
\hline HCV Ab: $-/+$ & $60.8 / 44.5$ & 0.39 & & \\
\hline Tumor size: $\leq 3 \mathrm{~cm} />3 \mathrm{~cm}$ & $54.7 / 51.8$ & 0.52 & & \\
\hline Tumor number: Single/multiple & $59.9 / 32.0$ & $<0.01$ & $2.27(1.16-4.36)$ & 0.02 \\
\hline Differentiation: Well/mod, poor & $78.8 / 47.8$ & 0.08 & & \\
\hline vv: $-/+$ & $54.2 / 51.5$ & 0.20 & & \\
\hline vp: $-/+$ & $54.2 / 51.5$ & 0.22 & & \\
\hline im: $-/+$ & $57.1 / 30.0$ & 0.06 & & \\
\hline PIVKAII: $\leq 400 />400$ & $57.5 / 44.2$ & $\mathbf{0 . 0 3}$ & $1.71(0.91-3.32)$ & 0.10 \\
\hline AFP: $\leq 200 />200$ & $58.9 / 51.5$ & 0.41 & & \\
\hline CA19-9: $\leq 37 />37$ & $57.3 / 35.7$ & 0.02 & $1.30(0.62-2.57)$ & 0.47 \\
\hline CEA: $\leq 5 />5$ & $66.5 / 16.0$ & $<0.01$ & $4.40(2.24-8.46)$ & $<0.01$ \\
\hline CEACAM1: Low/high & $58.1 / 29.7$ & 0.04 & $2.03(0.97-4.37)$ & 0.06 \\
\hline
\end{tabular}

vv: Venous invasion, vp: port vein invasion, im: intrahepatic metastasis, PIVKAII: protein induced by vitamin K absence or antagonists-II, AFP: alpha-feto-protein, CA19-9: carbohydrate antigen 19-9, CEA: Carcinoembryonic antigen, CEACAM1: carcinoembryonic antigen-related cell adhesion molecule 1, HR: hazard ratio, CI: confidence interval. Significant values are shown in bold.

\section{Acknowledgements}

Yuji Morine, Satoru Imura, Tetsuya Ikemoto, Jun Higashijima, Shuichi Iwahashi, Yu Saito, Chie Takasu, Shinichiro Yamada, Hiroki Teraoku, Atsushi Takata, Toshiaki Yoshimoto, and Mitsuo Shimada declare that no competing interests exist in regard to this study.

\section{References}

1 Llovet JM, Peña CE, Lathia CD, Shan M, Meinhardt G, Bruix J; SHARP Investigators Study Group: Plasma biomarkers as predictors of outcome in patients with advanced hepatocellular carcinoma. Clin Cancer Res 18: 2290-300, 2012.

2 Caraglia M, Giuberti G, Marra M, Addeo R, Montella L, Murolo M, Sperlongano P, Vincenzi B, Naviglio S, Prete SD, Abbruzzese A and Stiuso P: Oxidative stress and ERK1/2 phosphorylation as predictors of outcome in hepatocellular carcinoma patients treated with sorafenib plus octreotide LAR. Cell Death Dis 2: e150, 2011.

3 Gold P and Freedman SO: Specific carcinoembryonic antigens of the human digestive system. J Exp Med 122: 467-481, 1965

4 von Kleist S, Chavanel G, Burtin P: Identification of an antigen from normal human tissue that cross reacts with the carcinoembryonic antigen. Proc Natl Acad Sci USA 69: 2492-2494, 1972.

5 Sorokin JJ, Suquarbaker PH, Zamcheck N, Pisick M, Kupchik HZ and Moore FD: Serial carcinoembryonic antigen assays. Use in detection of cancer recurrence. JAMA 228: 49-53, 1974.

6 Gebauer F, Wicklein D, Horst J, Sundermann P, Maar H, Streichert T, Tachezy M, Izbicki JR, Bockhorn $M$ and Schumacher U: Carcinoembryonic antigen-related cell adhesion molecules (CEACAM) 1, 5 and 6 as biomarkers in pancreatic cancer. PLoS One 9(11): e113023, 2014.
Table III. Correlation between serum carcinoembryonic antigen (CEA) level and carcinoembryonic antigen-related cell adhesion molecule 1 (CEACAM1) expression.

\begin{tabular}{llcl}
\hline CEACAM1 & \multicolumn{2}{c}{ Serum CEA level } & \multirow{2}{*}{$p$-Value } \\
\cline { 2 - 3 } & Normal $(\mathrm{n}=99)$ & High $(\mathrm{n}=24)$ & \\
\hline Low & $56.7 \%(\mathrm{n}=56)$ & $12.5 \%(\mathrm{n}=3)$ & \multirow{20.01}{*}{} \\
High & $33.3 \%(\mathrm{n}=33)$ & $87.5 \%(\mathrm{n}=21)$ & \\
\hline
\end{tabular}

Significant values are shown in bold.

Table IV. Correlation between carcinoembryonic antigen-related cell adhesion molecule 1 (CEACAM1) and epithelial mesenchymal transition (EMT)-related factors and tumor angiogenesis.

\begin{tabular}{lccc}
\hline & \multicolumn{2}{c}{ CEACAM1 } & \multirow{2}{*}{-Value } \\
\cline { 2 - 3 } & Low $(\mathrm{n}=69)$ & High $(\mathrm{n}=54)$ & \\
\hline E-Cadherin & & & \\
Grade 1 $(\mathrm{n}=28)$ & $11 / 69(15.9 \%)$ & $17 / 54(31.5 \%)$ & \\
Grade 2 $(\mathrm{n}=64)$ & $36 / 69(52.2 \%)$ & $28 / 54(51.8 \%)$ & $<\mathbf{0 . 0 5}$ \\
Grade 3 $(\mathrm{n}=31)$ & $22 / 69(31.9 \%)$ & $9 / 54(16.7 \%)$ & \\
Vimentin & & & \\
Grade 1 $(\mathrm{n}=98)$ & $60 / 69(87.0 \%)$ & $38 / 54(70.4 \%)$ & 0.06 \\
Grade 2 $(\mathrm{n}=25)$ & $9 / 69(13.0 \%)$ & $16 / 54(29.6 \%)$ & \\
MVD & & $37.6 \pm 1.6$ & $<\mathbf{0 . 0 1}$ \\
Mean \pm SD (\%) & $28.2 \pm 2.2$ & 3
\end{tabular}

MVD: Microvessel density. Significant values are shown in bold. 
7 Scholzel, Zimmermann W, Schwarzkopf G, Grunert F, Rogaczewski B and Thompson J: Carcinoembryonic antigen family members CEACAM6 and CEACAM7 are differentially expressed in normal tissues and oppositely deregulated in hyperplastic colorectal polyps and early adenomas. Am J Pathol 156: 595-605, 2000.

8 Thom I, Schult-Kronefeld O and Byrkholder I, Schuch G, Andritky B, Kastendieck H, Edler L, Wagener C, Bokemeyer C, Schumacher $U$ and Laack E: Expression of CEACAM-1 in pulmonary adenocarcinomas and their metastases. Anticancer Res 29: 249-254, 2009.

9 Thies A, Moll I, Berger J, Wagener C, Brummer J, Schulze HJ, Brunner $G$ and Schumacher U: CEACAM1 expression in cutaneous malignant melanoma predicts the development of metastatic disease. J Clin Oncol 20: 2530-2536, 2002.

10 Xie S, Luca M, Huang S, Gutman M, Reich R, Johnson JP and Bar-Eli M: Expression of MCAM/MUC18 by human melanoma cells leads to increased tumor growth and metastasis. Cancer Res 57: 2295-2303, 1997.

11 Dango S, Sienel W, Schreiber M, Stremmel C, Kirschbaum A, Pantel $\mathrm{K}$ and Passlick B: Elevated expression of carcinoembryonic antigen-related cell adhesion molecule 1 (CEACAM$1)$ is associated with increased angiogenic potential in nonsmall-cell lung cancer. Lung Cancer 60: 426-433, 2008.

12 Duxbury MS: CEACAM1 cytoplastic expression is closely related to tumor angiogenesis and poorer relapse-free survival after curative resection of hepatocellular carcinoma. World J Surg 35: 2266-2267, 2011.

13 Kiriyama S, Yokoyama S, Ueno M, Haymi S, Ieda J, Yamamoto N, Yamaguchi S, Mitani Y, Nakamura Y, Tani M, Mishra L, Shively JE and Yamaue H: CEACAM1 long cytoplasmic domain isoform is associated with invasion and recurrence of hepatocellular carcinoma. Ann Surg Oncol 4: 505-514, 2014.

14 Sha QQ, Wei QZ, Zhu JK, Wang KX, Wang C, Liu HT, Yu WB, Li MX, Zhang GY: Loss of membranous carcinoembryonic antigenrelated cell adhesion molecule 1 expression is related to decreased relapse-free survival of hepatocellular carcinoma following liver transplantation. Chin Med J (Engl) 125: 2841-2845, 2012.

15 Dhanasekaran R, Nakamura I, Hu C, Chen G, Oseini AM, Seven ES, Miamen AG, Moser CD, Zhou W, van Kuppevelt TH, van Deursen JM, Mounajjed T, Fernandez-Zapico ME and Roberts LR: Activation of the TGF $\beta /$ SMAD transcriptional pathway underlies a novel tumor promoting role of sulfatase-1 in hepatocellular carcinoma. Hepatology 61: 1269-1283, 2015.

16 Liver Cancer Study Group of Japan (ed.): The General Rules for the Clinical and Pathological Study of Primary Liver Cancer. Tokyo: Kanehara; 2009.

17 Tanigawa N, Lu C, Mitsui T and Miura S: Quantitation of sinusoid-like vessels in hepatocellular carcinoma: its clinical and prognostic significance. Hepatology 26: 1216-1223, 1997.

18 Sun HC, Tang ZY, Li XM, Zhou YN, Sun BR and Ma ZC: Microvessel density of hepatocellular carcinoma: its relationship with prognosis. J Cancer Res Clin Oncol 125: 419-426, 1999.

19 Yamada S, Fuchs BC, Fujii T, Shimoyama Y, Sugimoto H, Nomoto S, Takeda S, Tanabe KK, Kodera Y and Nakao A: Epithelial-to-mesenchymal transition predicts prognosis of pancreatic cancer. Surgery 154: 946-954, 2013.

20 Murakami K, Kasajima A, Kawagishi N, Ohuchi N and Sasano $\mathrm{H}$ : Microvessel density in hepatocellular carcinoma: Prognostic significance and review of the previous published work. Hepatology Res 45: 1185-1194, 2015.
21 Thom I, Schult-Kronefeld O, Burkholder I, Schuch G, Andritzky B, Kastendieck H, Edler L, Wagener C, Bokemeyer C, Schumacher $\mathrm{U}$ and Laack E: Expression CEACAM-1 in pulmonary adenocarcinomas and their metastasis. Anticancer Res 29: 249-254, 2009.

22 Tovuu LO, Imura S, Utsunomiya T, Morine Y, Ikemoto, Arakawa Y, Mori H, Hanaoka J, Kanamoto M, Sugimoto K, Iwahashi S, Saito Y, Yamada S, Asanoma M, Miyake H and Shimada M: Role of CD44 expression in non-tumor tissue on intrahepatic recurrence of hepatocellular carcinoma. Int J Clin Oncol 18: 651-656, 2013.

23 Bruix and Sherman M: Management of hepatocellular carcinoma. Hepatology 42: 1208-1236, 2005.

24 Huppertz, Haraida S, Kraus A, Zech CJ, Scheidler J, Scheidler J, Breuer J, Helmberger TK and Reiser MF: Enhancement of focal liver lesions at gadoxetic acid-enhanced MR imaging: correlation with histopathologic findings and spiral CT--initial observations. Radiology 234: 468-478, 2005.

25 Bolondi L, Gaiani S, Celli N, Golfieri R, Grigioni WF, Leoni S, Venturi AM and Piscaglia F: Characterization of small nodules in cirrhosis by assessment of vascularity: the problem of hypovascular hepatocellular carcinoma. Hepatology 42: 27-34, 2005.

26 Gold P and Freedman SO: Demonstration of tumor specific antigens in human colonic carcinoma by immunologial tolerance and absorption technique. J Exp Med 1: 439-462, 1965.

27 Zhou H, Stanners CP and Fuks A: Specificity of anti-carcinoembryonic antigen monoclonal antibodies and their effects on CEA-mediated adhesion. Cancer Res 53: 3817-3822, 1993.

28 Saito Y, Shimada M, Utsunomiya T, Morine Y, Imura S, Ikemoto T, Mori H, Hanaoka J, Yamada S and Asanoma M: Prediction of recurrence of hepatocellular carcinoma after curative hepatectomy using preoperative Lens culinaris agglutinin-reactive fraction of alpha-fetoprotein. Hepatol Res 42: 887-894, 2012.

29 Hayashi M, Shimizu T, Hirokawa F, Inoue Y, Komeda K, Asakuma M, Miyamoto Y, Takeshita A, Shibayama Y and Tanigawa N: Clinicopathological risk factors for recurrence within one year after initial hepatectomy for hepatocellular carcinoma. Am Surg 77: 572-578, 2011.

30 Arabzadeh A, Dupaul-Chicoine J, Breton V, Haftchenary S, Yumeen S, Turbide C, Saleh M, McGregor K, Greenwood CM, Akavia UD, Blumberg RS, Gunning PT and Beauchemin N: Carcinoembryonic antigen cell adhesion molecule 1 long isoform modulates malignancy of poorly differentiated colon cancer cells. Gut 65(5): 821-829, 2015.

31 Ortenberg R, Galore-Haskel G, Greenberg I, Zamlin B, Sapoznik S, Greenberg E, Barshack I, Avivi C, Feiler Y, Zan-Bar I, Besser MJ, Azizi E, Eitan F, Schachter J and Markel G: CEACAM1 promotes melanoma cell growth through SOX-2. Neoplasia 16: 451-460, 2014.

32 Zhu J, Yang Y, Ma C, Zhang G, Wang K and Hu S: CEACAM1 cytoplastic expression is closely related to tumor angiogenesis and poorer relapse-free survival after curative resection of hepatocellular carcinoma. World J Surg 35: 2259-2265, 2011.

33 Bramswig KH, Pottler M, Unseld M, Wrba F, Uhrin P, Zimmermann W, Zielinski CC and Prager GW: Soluble carcinoembryonic antigen activates endothelial cells and tumor angiogenesis. Cancer Res 73: 6584-6596, 2013.

Received January 25, 2017

Revised February 26, 2017

Accepted February 26, 2017 